\title{
Article
}

\section{Factors that affect scrub practitioner non-technical skills: A literature review}

\author{
Mcclelland, Guy \\ Available at http://clok.uclan.ac.uk/22112/ \\ Mcclelland, Guy ORCID: 0000-0001-8053-1298 (2018) Factors that affect \\ scrub practitioner non-technical skills: A literature review. Journal of \\ Perioperative Practice, 28 (4). pp. 75-82. ISSN 2515-7949
}

It is advisable to refer to the publisher's version if you intend to cite from the work. http://dx.doi.org/10.1177/1750458918762315

For more information about UCLan's research in this area go to http://www.uclan.ac.uk/researchgroups/ and search for < name of research Group>.

For information about Research generally at UCLan please go to http://www.uclan.ac.uk/research/

All outputs in CLoK are protected by Intellectual Property Rights law, including Copyright law. Copyright, IPR and Moral Rights for the works on this site are retained by the individual authors and/or other copyright owners. Terms and conditions for use of this material are defined in the policies page.

\section{CLoK}

Central Lancashire online Knowledge www.clok.uclan.ac.uk

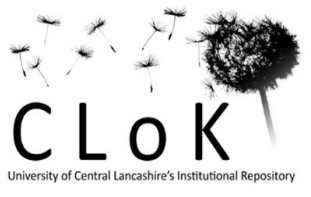




\title{
Factors that affect scrub practitioner non-technical skills: a literature
} review

\begin{abstract}
Non-technical skills are the cognitive and interpersonal behaviours that compliment clinical competence in surgery. Effective use of non-technical skills is essential for scrub practice because they facilitate anticipation of the surgeon's requirements and promote appropriate communication behaviours. This literature review analyses the determinants that may influence a scrub practitioner's use of non-technical skills during surgery. Recommendations are made that are intended to improve their use by reducing behavioural variations during surgery.
\end{abstract}

\section{Key words}

Non-technical skills, Patient Safety, Operating Theatre, Scrub Practitioner

\section{Background}

There is now acceptance that attainment of the optimal outcome for the surgical patient is dependent on the operating room team's competent use of conventional technical skills and their effective use of non-technical skills (NTS) (Sevdalis et al 2012). NTS are cognitive behaviours that include situational awareness and decision making and also interpersonal skills that include communication and teamwork (Flin et al 2008). Although the scrub practitioner's (SP) role during surgery requires the effective use of NTS to anticipate the surgeon's needs and timely communication to clarify any uncertainty, they are developed on a tacit basis (Mitchell \& Flin 2008). Consequently, the failure to recognise the influence of NTS on behaviours during surgery results in a high frequency of minor non-technical errors that require additional cognitive and interpersonal resources to prevent a serious untoward incident (Catchpole et al 2008). 
Effective use of NTS by SPs has been empirically measured as a contributory factor that improves the performance of the whole surgical team (McCulloch et al 2009 Morgan et al 2014b). However, scrub practitioners are frequently unable to consistently apply NTS effectively to compensate for other team members' deficits (Robertson et al 2014). This inability to apply NTS at a consistently effective level appears to have multiple causal factors that originate within the wider surgical team. Rather than the highly integrated level of teamwork one might expect to experience during surgery, Phitayahorn et al (2015) suggest that the outcomes each individual team member must accomplish result in practitioners working in isolation from their colleagues, despite sharing the same overarching aims. Scrub practitioners also possess lower baseline NTS capabilities when compared with their medical colleagues (Gillespie et al 2017) and these capabilities are further degraded when membership of surgical teams is inconsistent (Kang et al 2015).

The aim of this literature review is to analyse the determinants that affect the ability of SPs to consistently apply NTS effectively. Its specific objectives are to analyse the interpersonal, intrapersonal and environmental determinants that might affect SP NTS behaviours and how SPs respond to those determinants.

\section{Literature Search Strategies}

A literature search was undertaken in August 2015 using Medline and Cumulative Index Nursing and Allied Health Literature (CINAHL) Complete search engines. Medical Subject Headings (MeSH) and key terms were related to human factors, NTS, surgery and patient safety (see figure 1). The date limiter was set between 2004 and 2015. No other limiters were set. The search was updated in June 2016.

2502 titles and abstracts were reviewed. The primary screening inclusion criterion was primary research undertaken during the perioperative period. Secondary research that described the perioperative period was also included to add context to the background section. Primary and secondary research or articles addressing simulation, leadership and management, 
educational or therapeutic interventions were excluded at primary screening. Two potentially relevant papers were also excluded because they were unavailable in English. In total 2275 papers were excluded.

227 papers were reviewed during secondary screening. The secondary inclusion criteria were qualitative research that explored the societal context of surgery and quantitative observational research that measured SP NTS behaviours during surgery. Papers that described the intraoperative period but did not refer to the SP were excluded. Secondary research, empirical research on pre and post-op debriefings and questionnaire research were also excluded at this stage. 171 papers were excluded; 56 papers met the inclusion criteria. 44 papers were included in the final literature review after removal of duplications (see figure 1).

(Insert Fig. 1 about here)

\section{Results}

Three major themes emerged (see figure 2). These were;

- $\quad$ Naïve knowledge

- $\quad$ Techno-Social Context of Surgery

- $\quad$ Status asymmetry

(INSERT Fig. 2 about here)

\section{Naïve Knowledge}

SPS use NTS to facilitate their ability to respond to changing circumstances during surgery. Despite the fundamental nature of these skills, practitioners may not be able to explicitly state how they utilise NTS. Instead their use is derived from a tacit awareness of how to behave during surgery (Mitchell \& Flin 2008). Although tacit knowledge is a valid means of informing actions, its formation is dependent on an individual's previous exposure to a situation. 
Consequently, individuals may naively apply their interpretation of an appropriate reaction to situations and behaviours they experience (Foucault 1980). The literature review found that SPs re-constructed situations they had previously experienced to mediate effective reactions during surgery. This knowledge was found to be influenced by two determinants; tacit knowledge and memory based-knowledge.

\section{Tacit Knowledge}

The social environment of surgery appears to contribute to the accumulation of tacit knowledge of clinical situations that informs a practitioner's situational awareness. An ethnographic study conducted by Riley and Manias (2006) that explored time management during surgery suggested that tacit knowledge has limited practical use if individuals have limited experience of a specific surgeon or surgical procedure. Consequently, practitioners may prepare for surgery by recalling similar procedures they had previously experienced to inform their actions during forthcoming procedures.

The limitations of tacit knowledge of behaviours was emphasised in an interview study that explored the behavioural patterns of disruptive surgeons (Cochran \& Elder 2015). Practitioner self-efficacy was found to be eroded when surgeons displayed inappropriate behaviours. These behaviours resulted in reduced technical competence with a corresponding increase in errors. Rather than demonstrating a positive response to adversity, practitioners attempted to placate surgeons by becoming introverted (Cochran \& Elder 2015). Introversion reduced the SP's ability to influence behaviours that affect their practice, and the practice of others. Failure to develop an explicit awareness of the symbiotic relationship between appropriate behaviours in adversity, technical competence and self-efficacy isolates these conditions from each other and may perpetuate the use of inappropriate behaviours towards SPs by surgeons (Rogers et al 2011). 


\section{Memory-based knowledge}

SPs rely on memory-based knowledge to recall protocols that underpin practice. In a qualitative interview study that explored communication patterns, Gillespie et al (2010a) suggested that memory-based knowledge is essential because practitioners must apply the appropriate policy to the appropriate clinical situation whilst simultaneously monitoring their colleagues' compliance with policies. In a qualitative interview study that explored how surgical teams adapt to changing circumstances, participants suggested that memory-based knowledge is taught by supervising inexperienced practitioners and assigning appropriate tasks to their level of ability (Bogdanovic et al 2015). However, in the absence of a previous exposure to a similar procedure, cues that inform situational assessment may be missed or misinterpreted and result in an increase in the frequency of errors and omissions during instrument provision (Halverson et al 2011). Experienced practitioners are also fallible to this type of error. Gillespie et al (2009) found that when communication channels were unused, experienced practitioners encounter difficulty applying their existing knowledge to new procedures and surgeons. Team co-ordination was perceived as being disrupted, with a corresponding reduction in efficiency.

Memory-based knowledge affects NTS because its application is situational and dependent on exposure to previous similar experiences. Development of memory-based knowledge reduces the necessity to communicate because situational awareness develops an understanding of the team's objectives. However, the void that ensues in the absence of memory-based knowledge affects team co-ordination because communication channels are unused.

\section{Techno-Social Context of Surgery}

Surgical teams that are consistently staffed by the same team members allow SPs to become familiar with individual surgeons and develop an ability to interpret non-verbal indicators during surgery that complement technical competence. However, familiarity may also result in the 
establishment of social hierarchies that are not conducive for interprofessional working during surgery. Bjorn and Bostrom (2008) described these as informal "quasi-social" relationships. However, "quasi-professional socialisation" is a more appropriate descriptor because the literature review found that SPs were frequently excluded from communications by surgeons and only included when the surgeon perceived it to be appropriate.

\section{Technical competence}

A practitioner's position in relation to the surgeon's position improves their direction of gaze with corresponding improvements in their surgical activities. Korkiakangas et al (2014) found that subtle changes of instrument trolley positioning allowed closer observation of a surgeon's reactions. Subtle positioning changes also allowed experienced practitioners to prioritise observation of the surgical incision, whilst excluding potentially distracting aspects of the clinical environment $(p=<0.0001)$ (Koh et al 2011). Focussed observation increases situational awareness by allowing practitioners to pre-empt a surgeon's requirements. Zheng et al (2009) found that SPs with more than two years of experience provided a higher frequency of preemptive assistance than inexperienced colleagues $(p=0.008)$. Reactive assistance may result in increased inefficiencies because the novice practitioner has to interpret a verbal request, locate and then provide the instrumentation.

Accelerating the development of a novice's technical skills to improve the flow of surgical activity is regarded as a priority by surgeons and SPs. In a study that explored how postqualification education affected relationships with surgeons, Tame (2013) suggested that surgeons valued the development of a practitioner's technical skills because their functional outcomes were enhanced. Development of technical skills is prioritised because improved functional outcomes are visible; NTS development is a lesser priority because their contribution towards surgery is less tangible (Mitchell et al 2011). Achieving technical competence increases a practitioner's confidence to undertake assistance during increasingly complex surgical procedures. However, novice practitioners may then be unable to respond 
when surgical plans change unexpectedly because their technical competence may mask under-developed NTS processes (Bleakley et al 2013).

\section{Quasi-professional socialisation}

The literature review found that communication was frequently ineffective or even absent during surgery. An observational study of communication patterns during paediatric cardiac surgery found that surgeons frequently fail to inform scrub practitioners on the progress of surgery (Santos et al 2012). Consequently, situational awareness was diminished and compensatory behaviours from observing indicators in the clinical environment were required to ensure task completion. Observational studies have also found that attempts by SPs to obtain information directly from surgeons are frequently unsuccessful. In a study that explored how silence during surgery maintains existing social hierarchies by preserving the autonomy and authority of surgeons, Gardezi et al (2009) witnessed surgeons purposefully ignoring SPs' enquiries which resulted in unresolved uncertainty during surgical procedures. Other studies described how SPs developed alternative strategies to mitigate communication deficits. Gillespie et al (2013) found that information is gathered by eavesdropping on conversations between surgeons. The findings suggest that communication amongst individuals of an equal hierarchal status is easier to initiate than communication that has to overcome a steep hierarchal gradient. SPs have traditionally possessed a lower hierarchal status than their medical colleagues and this imbalance results in fewer opportunities to communicate. Consequently, strategies are initiated that facilitate development of shared situational awareness in the absence of effective communication.

Communication non-specific to surgery is a source of distraction. In a study that measured the frequency of case irrelevant communications (CICs) during surgery, Sevdalis et al (2007) observed that almost half of $\mathrm{CICs}$ are informal social conversations. The surgeon was found to be the main source $(p<0.01)$ and recipient $(p<0.001)$ of these conversations. Henrickson Parker et al (2012) observed that CICs are an attempt to diffuse tensions caused by surgery. 
Bjorn \& Bostrom (2008) suggested that this interaction is quasi-social because it does not attain the professional nature of a discussion about surgery. Although this strategy may be an attempt to rebuild team cohesion after a particularly stressful procedure, Bleakley et al (2013) observed that surgeons used these conversations as a strategy to introduce division between clinical staff and organisational management. The existing surgical hierarchy ensures that these discussions are socially coercive, rather than socially cohesive and that practitioners participate in these conversations to prevent becoming socially isolated (Higgins \& Maclntosh 2010). Quasi-professional socialisation during surgery may result in practitioners conforming with an existing culture where communication may be difficult to initiate, or is inappropriate because it is distracting or socially coercive. These conditions may increase the difficulty of communicating a practitioner's concerns when a patient safety concern arises.

\section{Status Asymmetry}

Bleakley et al (2013) described the concept of status asymmetry during surgery that resulted from the effect of professional hierarchies. These hierarchies result in individuals of lower hierarchal status being less able to influence decision making. The review suggested that these imbalances generated both intraprofessional and interprofessional dissonance.

\section{Intraprofessional Dissonance}

The review findings suggest that attitudes of experienced SPs towards novices affects intraprofessional relations by reducing team cohesion. Novices' clinical competence develops with assistance from their preceptors. Intraprofessional tensions originate from frustrations generated when preceptors perceived that preceptees were unable to apply their existing knowledge to new situations (Gillespie et al 2009). Preceptors may also withhold knowledge as a strategy that ensures the preceptee constructs their learning from negative experiences (Riley et al 2006). In an interview study by Rowland and Steeves (2010) that analysed the factors that contributed to incorrect surgical counts, one novice SP perceived that their preceptor's covert manipulation of these negative experiences affected the surgeon's 
perception of the novice's clinical competency. These findings suggest that preceptors may purposefully choose when to give or withhold appropriate clinical support to their novice colleagues and this choice may be based on the personal qualities of the mentor, rather than from assessment of the clinical situation.

\section{Inter-professional dissonance}

Surgical team members recognise that development of a team culture conducive to interprofessional collaboration during surgery is frequently difficult to attain (Undre et al 2006). Gillespie et al (2012) observed that when team membership was inconsistent, the resulting unfamiliarity contributed to increased frequency of miscommunications $(p<0.01)$. Consequently, the resulting surgical culture reduces collaboration and some surgeons may resort to the use of abrasive functional leadership styles to achieve their intended objectives (Rogers et al 2011). A qualitative interview study that explored the factors that constrain safety during surgery found that practitioners experienced increased levels of stress under these adverse conditions (Espin et al 2006). Consequently, surgical teams who continue to experience similar conditions develop strategies that act as buffers against the threat of error. Gillespie et al (2013) observed that SPs do this by developing an ability to judge the mood of the surgeon and then adjusting their behaviours correspondingly. However, this behaviour may cause the SP to reduce or withdraw communication in an attempt to prevent confrontation with surgeons (Henrickson Parker et al 2012).

\section{Discussion}

Ajzen's Theory of Planned Behaviour (1991) states that for past behaviour of a person to be an accurate predictor of their future behaviour, the determinants that affect their behaviour must remain unchanged. The findings of this review suggest the determinants that affect the use of NTS behaviours by SPs are multi-causal, dynamic in their origin and may be continually present in the surgical environment. Consequently, the NTS behaviours of practitioners may continue to be variable due to the unique context of each surgical procedure. Any changes to 
practice should aim to provide the conditions that reduce behavioural variation by SPs and their colleagues during surgical procedures. Interventions that could be considered include coaching of SPs and standardisation of practice and procedures during surgery (see fig. 3).

\section{Insert Fig. 3 about here}

Two research papers demonstrated that the intervention of the presence of team coach to facilitate team communication led to a significant improvement in post-test SP NTS performance (McCulloch et al 2009 Morgan et al 2014b). The presence of the team coach may have acted as a buffer by moderating the behaviours of the surgeon, whilst simultaneously empowering the SP to use available communication systems more effectively. However, the presence of professional team coaches is financially unsustainable for health organisations (Gillespie et al 2010b). The presence of a professional team coach may also cause a temporary "Hawthorne" effect of improved NTS behaviours that is not sustained after the team coach is withdrawn from the clinical area (Morgan et al 2014b). Alternatively, operating department managers should consider training experienced SPs to assume the visible role of "coach" to mentor and support inexperienced practitioners, or to provide support for experienced practitioners during complex surgical procedures. This role should also include the provision of constructive feedback to the practitioner on positive aspects of their performance, whilst highlighting areas for improvement. To enable this, operating departments should be prepared to invest in the development of practitioners in this role. The "coach" should receive training that allows that individual to develop explicit awareness of the contribution of NTS to clinical practice, and develop communication skills to feedback their observations of their colleagues' NTS behaviours.

In addition to the absence of formalised NTS training, there is often an absence of formalised training in the conduct of the swab count. This results in practitioners inconsistently applying swab count policies due to discrepant perceptions regarding who has ultimate responsibility for the process (D'Lima et al 2014). The National Safety Standards for Invasive Procedures 
(NatSSIPS) have been devised and published with the intention of reducing inconsistent application of fundamental safeguards during surgery and protect patients from unintentional harm (NHS England 2015). These standards recognise key stages during surgery that are vulnerable to task interruption, or behaviours that may compromise patient safety and are intended to improve inter-professional communication and increase situational awareness during surgery. However, many of these recommendations were already in place in the form of local policies (NHS England 2015). The inadequacies of local policies may just be one cause for error generation; the literature review suggests that the equilibrium between the factors that affect NTS is also a contributory cause of errors. NHS England (2015) suggests that existing policies and Standard Operating Procedures (SOPs) should be reviewed through a consultation process involving all professional groups involved in surgery prior to their implementation. However, the design of SOPs by practitioners using those standards may not necessarily lead to an increase in the compliance with the SOPs (Morgan et al 2014a). Organisations should introduce robust auditing to measure compliance with their Local Safety Standards for Invasive Procedures to ensure that all clinical staff involved with surgery comply with standards that are intended to improve their NTS.

The limitations of this literature review should be acknowledged. The literature search and theme formation was undertaken by a single researcher. The absence of collaboration may have resulted in selection bias that affected these processes. There are also limitations inherent in the retrieved literature that affects interpretation. Most of the research methodologies used observational methods to explore or measure the behaviours of the surgical team within the social context of surgery. Although SPs were explicitly referred to in the retrieved research papers, the inferences made of their behaviours were frequently secondary to the inferences made of surgeons' behaviours.

\section{Conclusion}


NTS behaviours permeate and influence all aspects of the SP's role. Despite this, their use remains tacit in nature. Future research should explore through ethnographic methodology how novice practitioners learn a tacit skill, and how experienced practitioners teach a tacit skill. Behavioural variations occur during surgery and these variations affect the engagement of the surgical team with standardised ways of working. Future studies should also use ethnographic methodology to explore the factors that affect standardisation of behaviours amongst surgical team members and how failure to standardise team behaviours affects SP NTS behaviours.

\section{References}

Ajzen I, 1991 The Theory of Planned Behaviour Organisational Behaviour and Human Decision making Processes 50 179-211

Bjorn C, Bostrom E, 2008 Theatre nurses understanding of their work: A phenomographic study at a hospital Journal of Advanced Perioperative Care 3 (4) 149-155

Bleakley A, Allard A, Hobbs, A 2013 "Achieving ensemble": communication in orthopaedic surgical teams and the development of situation awareness - an observational study using live videotaped examples Advances in Health Science Education 18 33-56.

Bogdanovic J, Perry J, Guggenheim M, Manser T 2015 Adaptive co-ordination in surgical teams BMC Health Services Research 15(128) 1-12

Catchpole K, Giddings A, Hirst G, Dale T, Peek G, de Leval M 2008 A method for measuring threats and errors in surgery Cognitive Technical Work Volume 10 pp 295-304 
Cochran A, Elder W 2015 Effects of disruptive surgeon behaviour in the operating room The American Journal of Surgery 209(1) pp 65-70

D'Lima D, Sacks M, Blackman W, Benn J (2014) Surgical swab counting: a qualitative analysis from the perspective of the scrub nurse Journal of Perioperative Practice 24 (5) 103-111

Espin S, Lingard L, Baker G, Regehr G 2006 Persistence of practice in everyday work: an exploration of organisational and psychological factors constraining safety in the operating room Quality Safety Health Care 15 165-170

Flin R, O'Connor P, Crichton M 2008 Safety at the Sharp End: a Guide to Non-Technical Skills. Farnham, Ashgate

Foucault M 1980 Power/Knowledge: Selected Interviews and Other Writings 1972-1977 Harlow, Pearson Education Limited

Gardezi F, Lingard L, Espin S, Whyte S, Orser B, Ross Baker G 2009 Silence, power and communication in the operating room Journal of Advanced Nursing 65(7) 1390-1399

Gillespie B, Chaboyer W, Fairweather N 2012 Interruptions and miscommunications in surgery Association of Operating Room Nurses' Journal 95 (5) 576-590.

Gillespie B, Chaboyer W, Longbottom P, Wallis M 2010a The impact of organisational factors on team communication in surgery: a qualitative study International Journal of Nursing Studies Volume $47 \mathrm{pp}$ 732-741

Gillespie B, Chaboyer W, Murray P 2010b Enhancing communication in surgery through team training interventions: a systematic literature review Association of Operating Room Nurses' Journal 92(6) $642-657$

Gillespie B, Chaboyer W, Wallis M, Chang HY, Werder H 2009 Operating theatre nurses' perception of competence: a focus group study Journal of Advanced Nursing 65 (5) 1019-1028 
Gillespie B, Gwinner K, Fairweather N, Chaboyer W 2013 Building shared situational awareness in surgery through distributed dialogue Journal of Multidisciplinary Healthcare 6 109-118

Gillespie B, Harbech E, Kang E, Steel C, Fairweather N, Chaboyer W 2017 Correlates of non-technical skills during surgery: a prospective study British Medical Journal Open 7 e014480 1-10

Halverson A, Casey J, Anderrson J et al 2011 Communication failure in the operating room. Surgery 149 (3) pp 305-310

Henrickson Parker S, Yule S, Flin R, McKinley A 2012. Surgeons' leadership in the operating room: an observational study The American Journal of Surgery (204) 347-354.

Higgins B, Maclntosh J 2010 Nurses' perceptions of the effects of physician perpetrated abuse. International Nursing Review 57 321-327

Kang E, Massey D, Gillespie B 2015 Factors that influence the non-technical skills performance scrub nurses: a prospective study Journal of Advanced Nursing 71(12) 2846-2857

Koh R, Park T, Wickens C, Ong LT, Chia SN 2011 Differences in attentional strategies by novice and experienced operating theatre scrub nurse Journal of Experimental Psychology: Applied 17(3) pp 233146

Korkiakangas T, Weldon S, Bezemer J, Kneebone R, 2014 Nurse-surgeon object transfer: Video analysis of communication and situation awareness in the operating theatre International Journal of Nursing Studies Volume 51 pp 1195-1206

McCulloch P, Mishra A, Handa A, Dale T, Hirst G, Catchpole K 2009 The effects of aviation style nontechnical skills training on technical performance and outcome in the operating theatre Quality Safe Health Care Volume 18, pp. 109-115.

Mitchell L, Flin R 2008 Non-technical skills of the operating theatre scrub nurse: literature review Journal of Advanced Nursing 63(1) pp 15-24 
Mitchell L, Flin R, Yule S, Mitchell J, Coutts K, Youngson G 2011 Thinking ahead of the surgeon: an interview study to identify scrub nurses' non-technical skills International Journal of Nursing Studies 48 818-828

Morgan L, New S, Robertson E et al 2014a Effectiveness of a facilitated introduction of a Standard Operating Procedure in the operating theatre: a controlled interrupted time series British Medical Journal Quality and Safety 24 120-127

Morgan L, Hadi M, Pickering S et al 2014b The effect of teamwork training on team performance and clinical outcome in elective orthopaedic surgery: a controlled interrupted time series British Medical Journal Open Access Volume 5 p. e006216

National Health Service England 2015 National Safety Standards for Invasive Procedures (NatSSIPS) [online] Available from https://www.england.nhs.uk/patientsafety/never-events/natssips/ [Accessed March 2017]

Phitayahorn R, Mineheart R, Hemingway M, Pian Smith MC, Petrusa E 2015 The relationship between intraoperative teamwork and management skills in patient care Surgery 158 (5) 1434-1440

Riley R, Manias E 2006 Governing time in operating rooms Issues in Clinical Nursing Volume 15 pp $546-553$

Riley R, Manias E, Polglase, A 2006 Governing the surgical count through communication interactions: implications for patient safety Quality Safety Healthcare 15 369-374

Robertson E, Hadi M, Morgan L et al 2014 Oxford NOTECHSII: a modified theatre team non-technical skills scoring system PLoS ONE 9(3) pp 1-8

Rogers D , Lingard L, Boehler $\mathrm{M}$ et al 2011 Teaching operating room conflict management to surgeons: clarifying the optimal approach Medical Education 45 939-945.

Rowlands A, Steeves R 2010 Incorrect surgical counts: a qualitative analysis Association of Operating Room Nurses Journal 92(4) 410-419 
Santos R, Bakero L, Franco P, Alves C, Fragata I, Fragata J 2012 Characterisation of non-technical skills in paediatric cardiac surgery: communication patterns European Journal of Cardio-Thoracic Surgery 41 1005-1012

Sevdalis N, Healey N, Vincent C 2007 Distracting communications in the operating theatre Journal of Evaluation in Clinical Practice 13 390-394

Sevdalis N, Hull L, Birnbach DJ 2012 Improving patient safety in the operating theatre and perioperative care: obstacles, interventions, and priorities for accelerating progress British Journal of Anaesthesia Volume 109 (s1) i3-i16

Tame S, 2013 The effect of continuing professional education on perioperative nurses' relationships with medical staff: findings from a qualitative study Journal of Advanced Nursing 69(4) 817-827

Undre S, Sevdalis N, Healey A, Darzi S, Vincent C 2006 Teamwork in the operating theatre: cohesion or confusion? Journal of Evaluation in Clinical Practice 12(2)182-189

Zheng B, Taylor M, Swanstrom L 2009 An obsservational study of surgery-related activities between nurses and surgeons during laparoscopic surgery The American Journal of Surgery Volume $197 \mathrm{pp}$ 497-502 


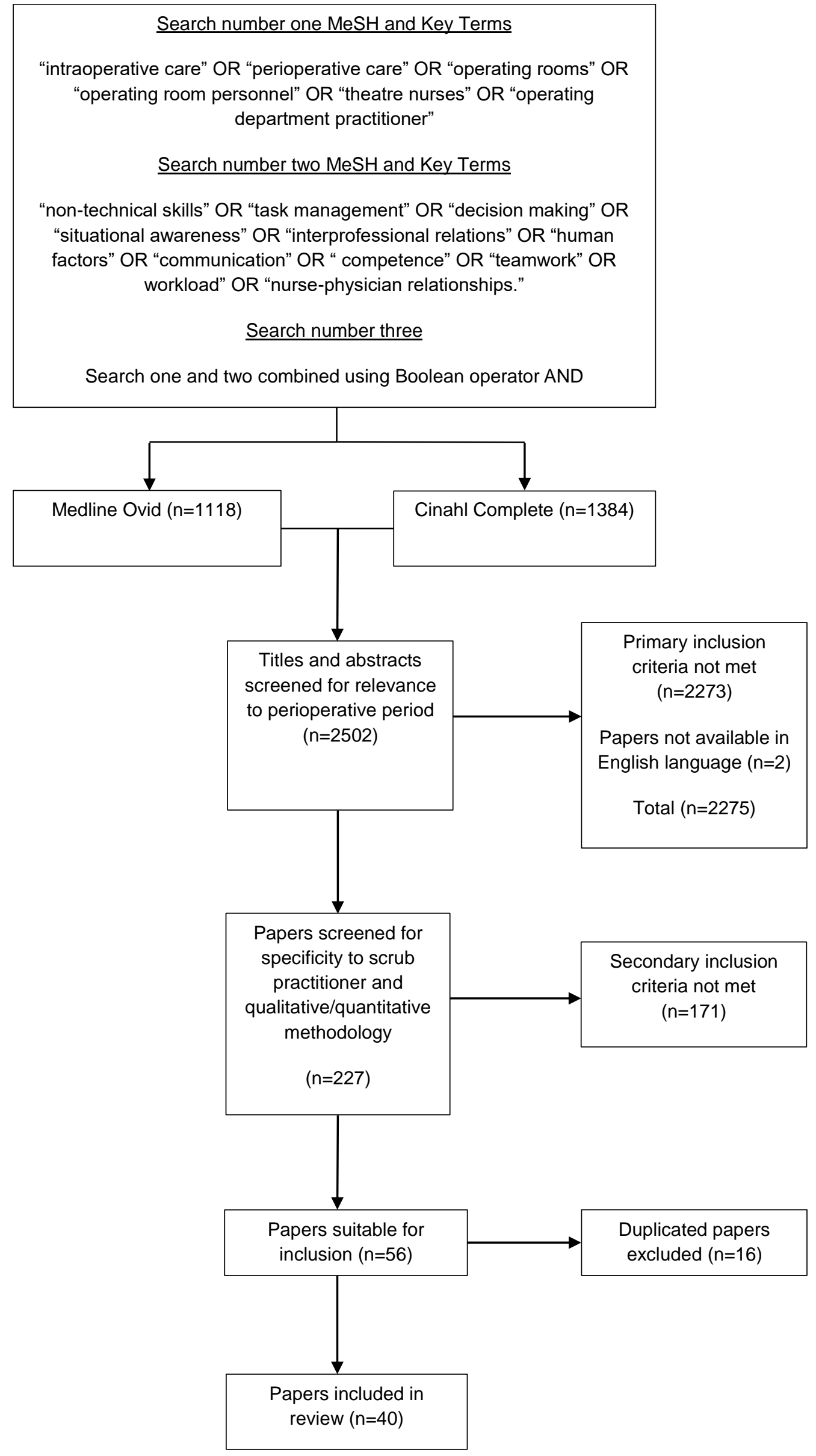




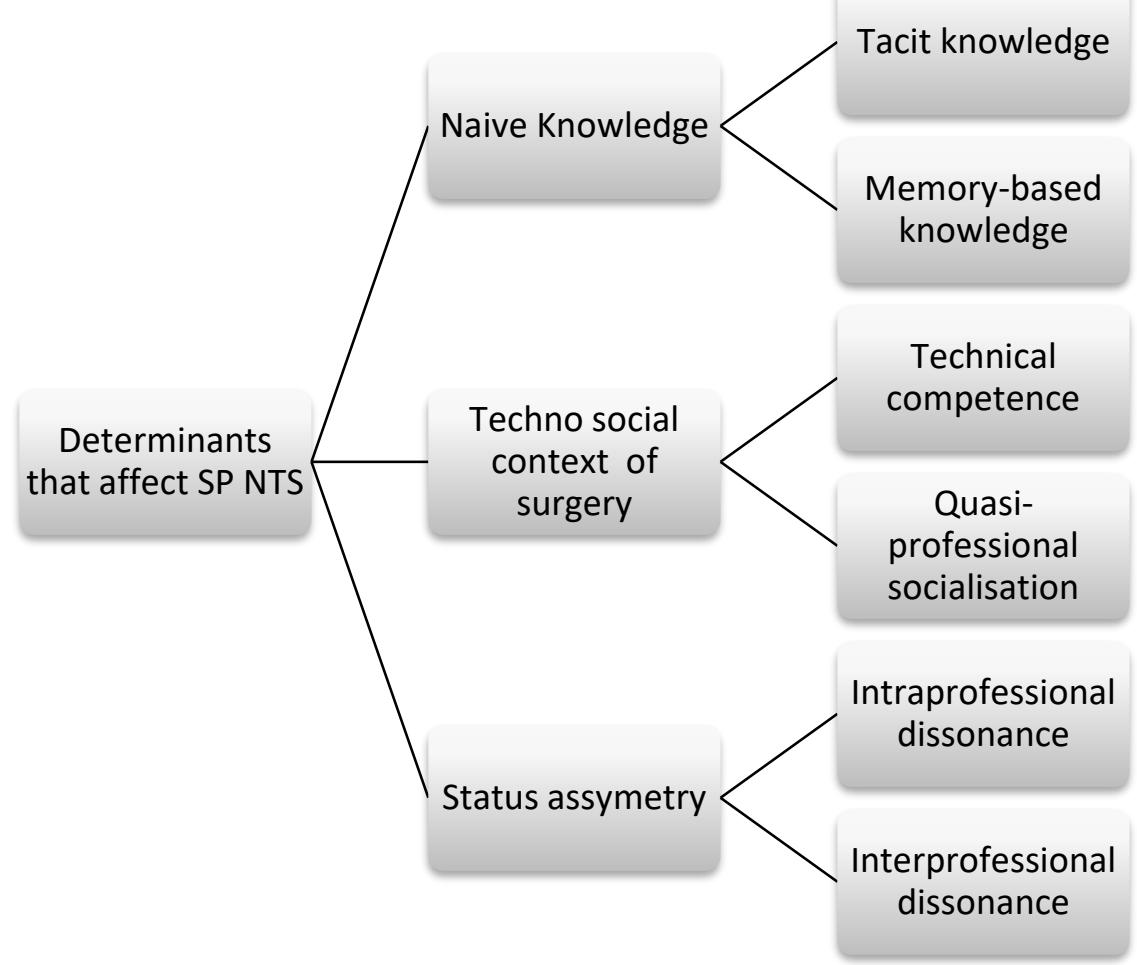

Figure 3 -Reducing variability in the use of NTS by SPs during surgery

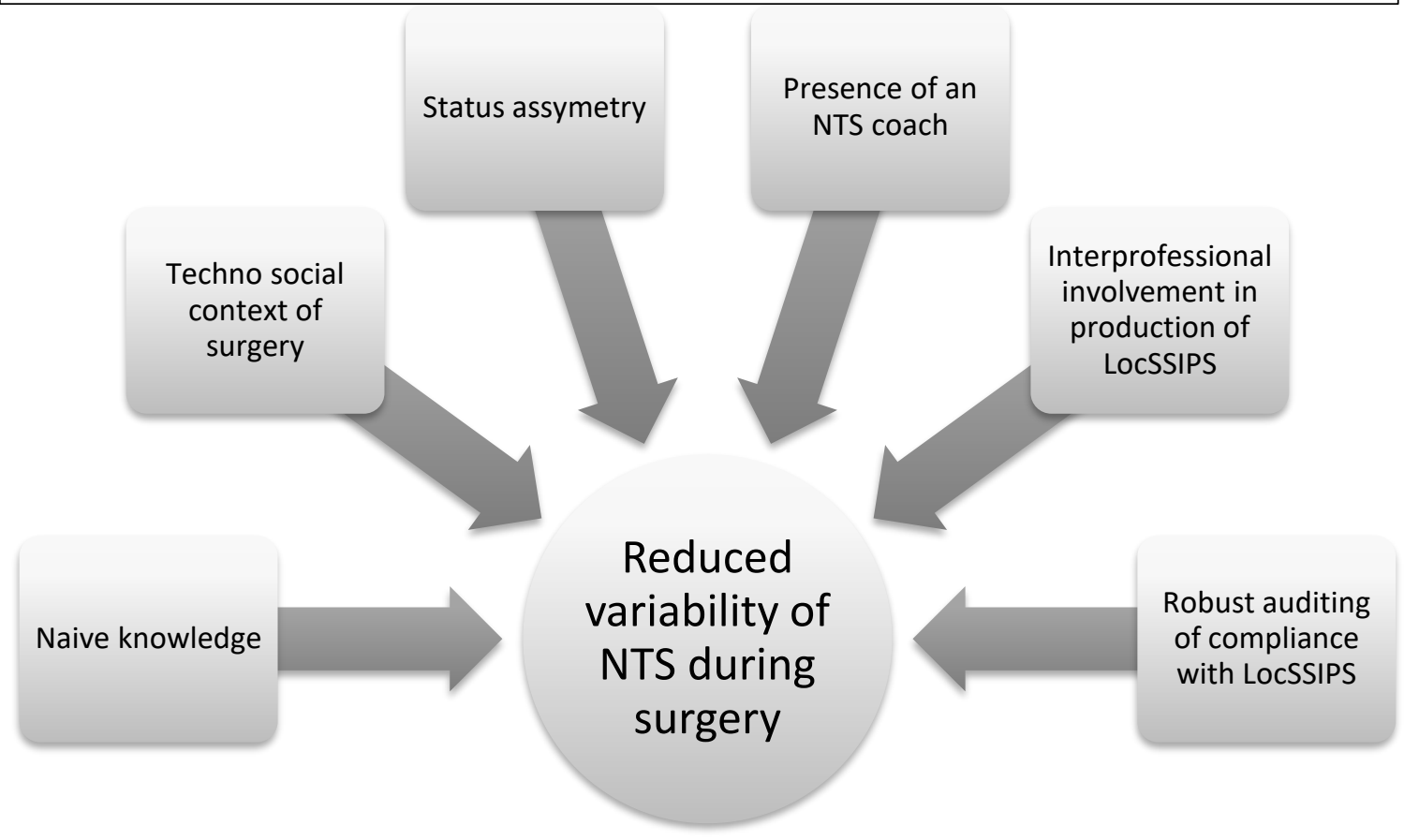

